\title{
A bisection algorithm for grammar- based compression of ordered trees
}

\author{
$\operatorname{AUTHOR}(\mathrm{S})$ :
}

Akutsu, Tatsuya

\section{CITATION:}

Akutsu, Tatsuya. A bisection algorithm for grammar-based compression of ordered trees. Information Processing Letters 2010, 110(18-19): 815820

\section{ISSUE DATE:}

2010-09-15

URL:

http://hdl.handle.net/2433/139421

\section{RIGHT:}

C 2010 Elsevier B.V.; この論文は出版社版でありません。引用の際には 出版社版をご確認ご利用ください。; This is not the published version. Please cite only the published version. 


\title{
A bisection algorithm for grammar-based compression of ordered trees
}

\author{
Tatsuya Akutsu \\ Bioinformatics Center, Institute for Chemical Research, Kyoto University, \\ Uji, Kyoto 611-0011, Japan. \\ e-mail: takutsu@kuicr.kyoto-u.ac.jp
}

keywords: approximation algorithms, graph algorithms, tree grammars, data compression

\section{Introduction}

Among various approaches to data compression for text data, extensive studies have been done on grammar-based compression. Grammar-based compression is to find a small grammar generating a given string, and is useful not only for data compression but also for pattern extraction. From a theoretical viewpoint, it is known that finding the smallest context-free grammar (CFG) is NP-hard but it is approximated in polynomial time within a factor of $O\left(\log \left(n / m^{*}\right)\right)$, where $n$ is the size of an input data and $m^{*}$ is the size of the smallest gram$\operatorname{mar}[3,6,8]$.

It is reasonable to try to extend grammar-based compression for tree structured data. Indeed, various grammars and algorithms have been developed for that purpose [2, 7, 9]. However, to my knowledge, no algorithm has been known with a guaranteed approximation ratio. In this paper, we mainly consider rooted ordered trees. We define an elementary ordered tree grammar (EOTG) by extending CFG, and then present a polynomial time algorithm which approximates the smallest EOTG within a factor of $O\left(n^{5 / 6}\right)$. We also show that the grammar and algorithm can be modified for rooted unordered trees of bounded degree.

\section{Preliminaries}

In this paper, we consider rooted ordered trees unless otherwise stated. For a tree $T, V(T)$, $E(T)$ and $r(T)$ denote a set of nodes, a set of edges and the root of $T$, respectively. The size of $T$ is the number of nodes in $T$ and is denoted by $|T|$. For a node $v$ in a tree $T, T(v)$ denotes the subtree of $T$ induced by $v$ and its descendants. $T-T(v)$ and $T-T(v) \cup\{v\}$ denote 
the subtrees of $T$ induced by $V(T)-V(T(v))$ and by $V(T)-V(T(v)) \cup\{v\}$, respectively.

The depth of a node $v$ is the number of edges in the path from the root to $v$ and is denoted by $d(v) . \quad V_{d}(T)$ denotes the set of nodes of depth $d$ in a tree $T$. For a node $u$ and its children $v_{1}, \ldots, v_{g}, \operatorname{sub}\left(u, v_{i_{1}}, \ldots, v_{i_{h}}\right)$ denotes the subtree induced by $u, v_{i_{1}}, \ldots, v_{i_{h}}$ and the descendants of $v_{i_{1}}, \ldots, v_{i_{h}}$, where $\left(v_{i_{1}}, \ldots, v_{i_{h}}\right)$ is a subsequence of $\left(v_{1}, \ldots, v_{g}\right)$. For a string $s, s[i]$ and $s[i, j]$ denote the $i$ th letter of $s$ and a substring between the $i$ th and $j$ th positions of $s$, respectively.

For simplicity, we treat each tree $T$ as an edge labeled tree and let $\Sigma$ be the set of edge labels. Node labeled trees can be transformed into edge labeled trees by assigning a label of a node (except the root) to the edge between the node and its parent. The depth-first search traversal of $T$ (i.e., visiting children of each node according to their left-to-right order) gives an Euler tour beginning from the root and ending at the root where each edge $\{w, v\}$ is traversed twice in the opposite directions. Let $\Sigma^{\prime}=\{a, \bar{a} \mid a \in \Sigma\}$, where $\bar{a} \notin \Sigma$. Let $\left(e_{1}, e_{2}, \ldots, e_{2 n-2}\right)$ be the sequence of directed edges in the Euler tour of $T$ of size $n$. From this, we create the Euler string es $(T)$ of length $2 n-2$ over $\Sigma^{\prime}$. Let $e=\{u, v\}$ be an edge in $T$, where $u$ is the parent of $v$. Suppose that $e_{i}=(u, v)$ and $e_{j}=(v, u)$. It is to be noted that $i<j$ holds since $e_{i}$ s are ordered according to the Euler tour of $T$. Then, we define $i_{1}(e)$ and $i_{2}(e)$ by $i_{1}(e)=i$ and $i_{2}(e)=j$, respectively. We define es $(T)$ by letting $e s(T)\left[i_{1}(e)\right]=\ell(e)$ and $e s(T)\left[i_{2}(e)\right]=\overline{\ell(e)}$, where $\ell(e)$ is the label of $e$. It is known that $T_{1}$ is isomorphic to $T_{2}$ (including label information) if and only if $e s\left(T_{1}\right)=e s\left(T_{2}\right)[1]$.

Of course, we can apply existing grammar-based string compression algorithms to Euler strings in order to compress trees. Since our tree grammars can be transformed into CFGs as shown in the proof of Lemma 2, such an approach may yield better compression performances. However, in such a case, derived grammars do not necessarily correspond to tree grammars. As discussed in $[7,9]$, the purpose of grammar-based tree compression is not only to compress input trees but also to extract features (e.g., patterns) from input trees. Therefore, we need to obtain tree grammars from input trees.

\section{Elementary Ordered Tree Grammar}

We consider two types of trees: tagged trees and non-tagged trees. A non-tagged tree is a usual tree, where either a terminal symbol or a nonterminal symbol is attached as a label to each edge. A tagged tree is the same as a non-tagged tree except that exactly one leaf node is 
tagged. An edge whose lower endpoint is a tagged node is called a tagged edge. The restriction of the number of tagged nodes is important. If multiple tagged nodes were allowed per tree, the resulting grammars would become more complicated and the time complexity of parsing would become much higher because we might examine much more numbers of combinations in parsing. Furthermore, it is unclear whether we can design a compression algorithm with a guaranteed approximation ratio because our proposed algorithm heavily depends on the fact that there exists at most one tagged node in a tree. We use a capital letter to denote a nonterminal symbol and a lower-case letter to denote a terminal symbol. We may identify an edge with its label, and a tree with its Euler string.

We consider the following two types of production rules for trees (see also Fig. 1)

(R1) Replace a non-tagged edge by a non-tagged tree $T$,

(R2) Replace a tagged edge by a tagged tree $T_{x}$,

where we start with a tree consisting of a non-tagged edge with the start symbol $S$.

For a tagged tree $T_{x}$, es $\left(T_{x}\right)$ also denotes the Euler string of $T_{x}$ except that the tagged edge with label $A$ in $T_{x}$ is transformed into $A x \bar{A}$, where $x$ is the special symbol denoting the tag. We have an Euler string version of production rules as: (R1') $A \bar{A} \rightarrow$ es(T), (R2') $A x \bar{A} \rightarrow e s\left(T_{x}\right)$. The size of a grammar is defined as the total number of letters (in Euler strings) appearing in the right hand sides (RHSs) of rules excluding the tag symbol. That is, the size is the double of the number of edges in trees appearing in RHSs. An EOTG is defined by a 4 -tuple $(\Sigma, \Gamma, S, \Delta)$ where $\Sigma, \Gamma, S \in \Gamma$ and $\Delta$ are a set of terminal symbols, a set of nonterminal symbols, the start symbol and a set of production rules, respectively. When we discuss compression algorithms, as in [3], we only consider EOTGs satisfying (i) each nonterminal appears in LHS of exactly one rule, and (ii) there exists an ordering of the nonterminals $\Gamma$ such that each nonterminal precedes all nonterminals in its definition (i.e., the grammar is acyclic). Due to these properties, it is guaranteed that a grammar generates exactly one finite-size tree.

In this paper, we consider a special class of EOTG in which only the following types of production rules are allowed

$$
\begin{aligned}
& \text { (I) } A \bar{A} \rightarrow a \bar{a}, \quad\left(\text { I'}^{\prime}\right) A x \bar{A} \rightarrow a x \bar{a}, \quad(\mathbf{I I}) A \bar{A} \rightarrow B C \overline{C B}, \quad\left(\mathbf{I I}^{\prime}\right) A x \bar{A} \rightarrow B C x \overline{C B}, \\
& \text { (III) } A \bar{A} \rightarrow B \bar{B} C \bar{C}, \quad \text { (IIIA) } A x \bar{A} \rightarrow B \bar{B} C x \bar{C}, \quad \text { (IIIB) } A x \bar{A} \rightarrow B x \bar{B} C \bar{C} .
\end{aligned}
$$


(A)
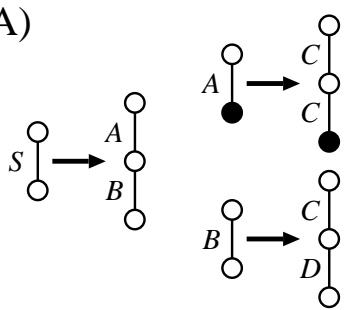

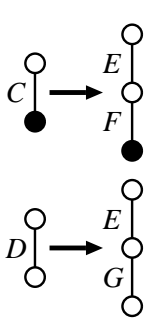

(B)
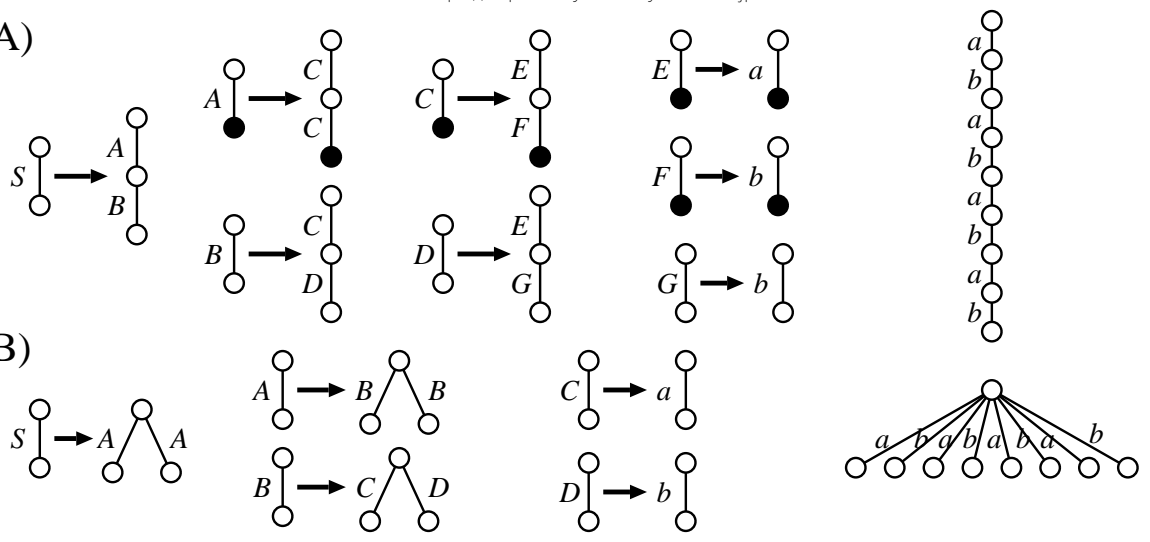

(C)
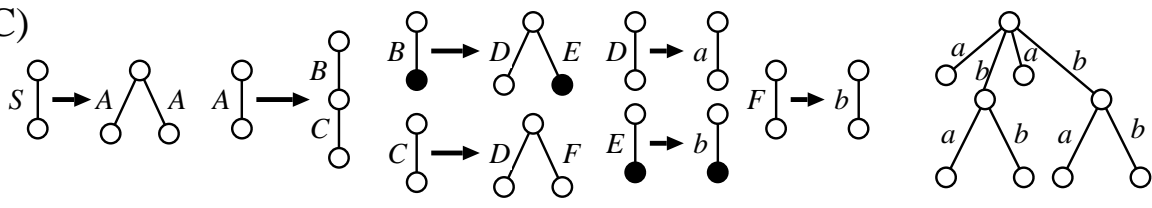

Figure 1: Examples of SEOTGs (left) and generated trees (right). Black nodes denote tagged nodes.

This restricted version of EOTG is referred as SEOTG (Simple EOTG). From examples (A) and (B) of Fig. 1, it is seen that EOTG is an extension of CFG for both vertical and horizontal directions. Though the number of rules of grammar (A) is greater than that for CFG (because of handling of tags), it is at most double.

Lemma 1 Any EOTG of size $m$ can be transformed into an SEOTG of size at most $3 m$ that generates the same set of trees as EOTG does.

Proof. We only show a recursive procedure to transform $A \rightarrow T_{x}$, where $T_{x}$ is a tagged tree of size at least 3. Then, it is straight-forward to extend the procedure for all cases.

If there is only one child $v$ of $r=r\left(T_{x}\right)$, we add $A x \bar{A} \rightarrow B C x \overline{C B}$, where $B$ and $C$ are nonterminal edges for generating an edge $(r, v)$ and a subtree rooted at $v$, respectively. Otherwise, suppose that there are $g$ children $v_{1}, \ldots, v_{g}$ of $r$. If $T_{x}\left(v_{1}\right)$ is a tagged tree, we add $A x \bar{A} \rightarrow B x \bar{B} C \bar{C}$, otherwise we add $A x \bar{A} \rightarrow B \bar{B} C x \bar{C}$, where $B$ and $C$ correspond to $\operatorname{sub}\left(r, v_{1}\right)$ and $\operatorname{sub}\left(r, v_{2}, \ldots, v_{g}\right)$, respectively.

Suppose that $T_{x}$ consists of $m^{\prime}$ edges. Then, the corresponding EOTG rule has size $2 m^{\prime}$. The number of production rules of the former type (including rules without tags) generated from $T_{x}$ is bounded by $m^{\prime}$ because there exist at most $m^{\prime}$ edges in $T_{x}$ labeled with nonterminal symbols. The number of production rules of the latter type is bounded by $m^{\prime}-1$ because 
there exist at most $m^{\prime}$ edges in $T_{x}$, and each production rule partitions a relevant set of edges into two disjoint sets of edges ${ }^{1}$. Therefore, the total size of the resulting production rules for $T_{x}$ is bounded by $2 m^{\prime}+4\left(m^{\prime}-1\right)=6 m^{\prime}-4$, which is smaller than $3 \times 2 m^{\prime}$. By summing the sizes of all resulting rules, we have the lemma.

\section{Parsing Algorithm}

Before discussing the compression algorithm, we show that parsing of a string for any EOTG (including ambiguous and cyclic cases) can be done in polynomial time using a dynamic programming (DP) algorithm. Based on Lemma 1, we only present an algorithm for SEOTG. For each nonterminal symbol $A$ for rules of type (I'), (II'), (IIIA) and (IIIB) (resp. type (I), (II) and (III)), we construct a table $A[i, h, k, j]$ (resp. $A[i, j]$ ) where $i \leq h \leq k \leq j$. $A[i, h, k, j]=1$ if $\operatorname{es}(T)[i, h]$ and $\operatorname{es}(T)[k, j]$ are derived from $A x \bar{A}$, where the concatenation of $e s(T)[i, h]$ and $e s(T)[k, j]$ corresponds to a subtree, and $e s(T)[h+1, k-1]$ corresponds to a subtree rooted at the lower endpoint of an edge corresponding to $e s(T)[h]$ and $e s(T)[k]$. Suppose that RHS of $A x \bar{A}$ is of type (II'). Then, $A[i, h, k, j]$ can be computed by the following DP procedure

$$
A[i, h, k, j]= \begin{cases}1 & \text { if }(\exists g, f)(B[i, g, f, j]=1 \text { and } C[g+1, h, k, f-1]=1), \\ 0 & \text { otherwise. }\end{cases}
$$

For other type rules, $A[i, h, k, j]$ (or $A[i, j]$ ) can also be computed in a similar way. Since the size of $A[i, h, k, j] \mathrm{s}$ is $O\left(m n^{4}\right)$ and the time required per entry is $O\left(n^{2}\right)$ where $m$ is the size of EOTG and $n=|T|$, the following theorem holds.

Theorem 1 Whether or not a given tree $T$ is generated from a given EOTG can be decided in $O\left(m n^{6}\right)$ time.

\section{Compression Algorithm}

The compression algorithm is based on BISECTION $[3,5]$ and is denoted by TREE-BISECTION here. TREE-BISECTION recursively decomposes a given tree $T_{0}$ into smaller subtrees (see Fig. 2) until each subtree consists of an edge.

As a base case, suppose that the current tree $T$ consists of an edge with label $a$. Then, we add the rule of $A x \bar{A} \rightarrow a x \bar{a}$ if $T$ is a tagged tree, and $A \bar{A} \rightarrow a \bar{a}$ otherwise.

\footnotetext{
${ }^{1}$ This property can be seen from the fact that every binary tree with $m^{\prime}$ leaves has $m^{\prime}-1$ internal nodes, where leaves correspond to edges in $T_{x}$ and internal nodes correspond to production rules.
} 
Next, suppose that $T$ is a non-tagged tree of size greater than 2. Let $r$ be the root of $T$. Let $u_{1}, \ldots, u_{h}$ be the children of a node $u$. Then, $u_{j}$ is called the heaviest child (among $\left.u_{1}, \ldots, u_{h}\right)$ if $\left|T\left(u_{j}\right)\right|$ is largest. Let $\left(v_{0}, v_{1}, v_{2}, \ldots, v_{g}\right)$ be a heavy chain of $T$, which is constructed by following the heaviest children from the root $v_{0}=r$. Let $v_{i}$ be the first node such that $\left|T\left(v_{i}\right)\right| \leq \frac{1}{2}|T|$. Here we let $v=v_{i-1}$. We partition $T$ into $T_{1}=T-T(v) \cup\{v\}$ and $T_{2}=T(v)$, where $T_{1}$ becomes a tree with tagged $v$, and $\left|T_{1}\right| \leq \frac{1}{2}|T|+1$. Let $w_{1}, \ldots, w_{h}$ be the children of $v$. Then, $\left|T\left(w_{l}\right)\right| \leq \frac{1}{2}|T|$ holds for all $w_{l}$. Next, we find $w_{j}$ that minimizes

$$
|| \operatorname{sub}\left(v, w_{1}, \ldots, w_{j}\right)|-| \operatorname{sub}\left(v, w_{j+1}, \ldots, w_{h}\right)||,
$$

where the tie is broken arbitrarily. Then, we partition $T_{2}$ into $T_{3}=\operatorname{sub}\left(v, w_{1}, \ldots, w_{j}\right)$ and $T_{4}=\operatorname{sub}\left(v, w_{j+1}, \ldots, w_{h}\right)$. From $\left|T\left(w_{l}\right)\right| \leq \frac{1}{2}|T|$ for all $w_{l}$, we can see that $\left|T_{3}\right| \leq \frac{3}{4}|T|+1$ and $\left|T_{4}\right| \leq \frac{3}{4}|T|+1$ hold. The associated rules of SEOTG are created accordingly (see Fig. 2 $(\mathrm{A}))$.

Finally, suppose that $T$ is a tagged tree of size greater than 2. Let $r$ be the root of $T$. Let $\left(v_{0}=r, v_{1}, v_{2}, \ldots, v_{g}=x\right)$ be the path from the root to the tagged node $x$. Let $v_{i}$ be the first node in the path such that $\left|T\left(v_{i}\right)\right| \leq \frac{1}{2}|T|$. Here we let $v=v_{i-1}$. As in the case of non-tagged tree, we partition $T$ into $T_{1}=T-T(v) \cup\{v\}$ and $T_{2}=T(v)$. Let $w_{1}, \ldots, w_{h}$ be the children of $v$, and $w_{j}=v_{i}$. Then, we partition $T_{2}$ into $T_{3}=\operatorname{sub}\left(v, w_{1}, \ldots, w_{j}\right)$ and $T_{4}=\operatorname{sub}\left(v, w_{j+1}, \ldots, w_{h}\right)$ if $\left|\operatorname{sub}\left(v, w_{1}, \ldots, w_{j}\right)\right|<\left|s u b\left(v, w_{j}, \ldots, w_{h}\right)\right|$. Otherwise, we partition $T_{2}$ into $T_{3}=\operatorname{sub}\left(v, w_{1}, \ldots, w_{j-1}\right)$ and $T_{4}=s u b\left(v, w_{j}, \ldots, w_{h}\right)$. Here, we assume without loss of generality that $T_{4}$ contains $w_{j}$. From $\left|T_{4}\right| \leq\left|T_{3}\right|$, we can see that $\left|T_{4}\right| \leq \frac{1}{2}|T|+1$ holds. Though the size of $T_{3}$ may be close to $|T|, T_{3}$ is a non-tagged tree and thus is decomposed into subtrees whose sizes are not greater than $\frac{3}{4}|T|$ in the next recursive step. The associated rules of SEOTG are created accordingly (see Fig. 2 (B)). It is to be noted that each of $T_{1}, T_{2}, T_{3}$ and $T_{4}$ contains at most one tagged node.

There are some exceptional cases: either $T_{1}$ is empty or $v_{i-1}$ has only one child. In the former case, we directly decompose $T$ into $T_{3}$ and $T_{4}$. In the latter case, we directly decompose $T$ into $T_{1}$ and $T_{2}$. In each case, the properties on the size of trees and the number of tagged nodes are preserved.

If a generated subtree $T$ is isomorphic to a previously generated subtree $T^{\prime}$, we assign the same nonterminal label to both edges corresponding to $T$ and $T^{\prime}$, and do not recur for further decomposition of $T$. 

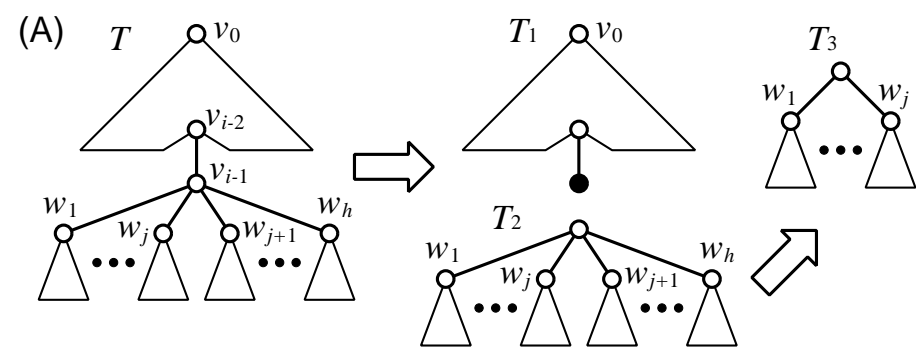

$\overbrace{}^{T_{4}} \overbrace{}^{w_{h}}$

$A_{0} \rightarrow \prod_{0}^{A_{1}}$

$A_{2} \jmath_{0} \rightarrow A_{3} \overbrace{0}^{A_{4}}$

(B)
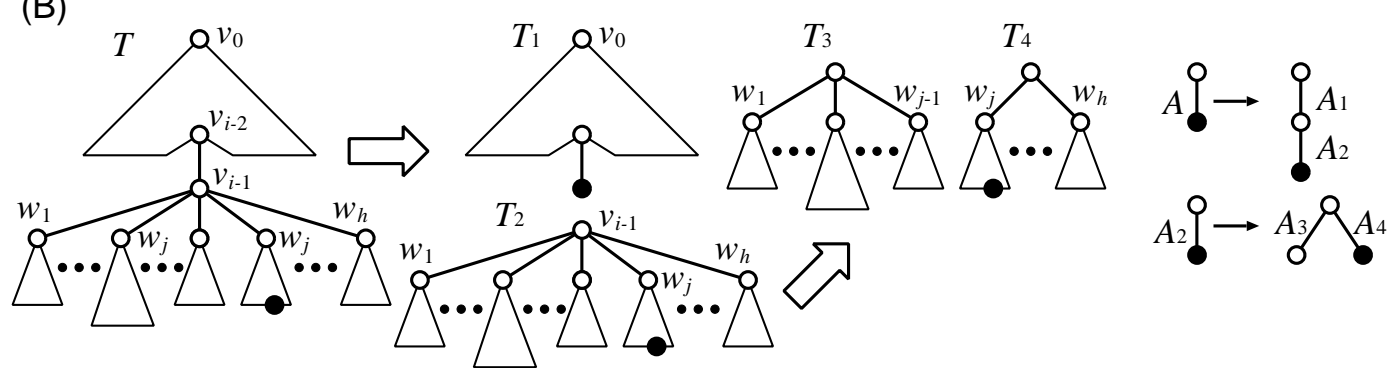

Figure 2: Illustration of TREE-BISECTION. (A) Case of non-tagged tree $T$. (B) Case of tagged-tree $T$. In each case, $T_{i}$ is generated from $A_{i}$.

\section{Analysis}

If we consider trees of height 1 (i.e., the depth of each node is at most 1), EOTG corresponds to CFG and thus the lower bounds on the approximation ratio on compression in [3] holds for EOTG. In the same way, the lower bound for BISECTION (Theorem 5 in [3]) holds also for TREE-BISECTION.

Proposition 1 The approximation ratio of TREE-BISECTION is $\Omega(\sqrt{n} / \log n)$.

In order to analyze the upper bound of TREE-BISECTION, we first establish $m k$ Lemma [3] for EOTG.

Lemma 2 If a tree $T$ is generated by an EOTG of size $m$, es $(T)$ contains at most $2 m k$ distinct substrings of length $k$.

Proof. We transform EOTG into CFG by splitting each rule (except the starting one) into a pair of rules in CFG by breaking LHS and RHS of each rule before and after $x$. For example, $A x \bar{A} \rightarrow B \bar{B} C x \bar{C}$ is split into $A \rightarrow B \bar{B} C$ and $\bar{A} \rightarrow \bar{C}$. Since we assume that a non-empty unique Euler string is generated by a given EOTG, the same string is generated by this CFG of size $2 m$. Then, the proof of $m k$ Lemma in [3] can be directly applied to this case. 
Using the above mentioned relationship between EOTG and CFG, the following proposition directly follows from Lemma 1 of [3].

Proposition 2 The smallest EOTG that generates a tree of size $n$ has size $\Omega(\log n)$.

Here, we consider the tree $\mathcal{T}$ representing a recursive process of TREE-BISECTION, where each subtree constructed in TREE-BISECTION is associated with a distinct node in $\mathcal{T}$ in the following way. $T_{0}$ corresponds to the root of $\mathcal{T}$. If $T$ is decomposed into $T_{1}$ and $T_{2}$ in TREEBISECTION, the node corresponding to $T$ has two children corresponding to $T_{1}$ and $T_{2}$. For each node $p$ of $\mathcal{T}, e(p)$ denotes the number of edges in the corresponding tree.

Lemma $3 \sum_{p \in V_{d}(\mathcal{T})} e(p) \leq\left|E\left(T_{0}\right)\right|=n-1$ holds for any $d$.

Proof. TREE-BISECTION recursively decomposes a tree into edge disjoint subtrees. Since the sum is taken over edge disjoint subtrees that are obtained from $T_{0}$, the lemma holds.

Lemma 4 The depth of recursive calls of TREE-BISECTION is $O(\log n)$.

Proof. Consider any downward path $\left(p_{1}, p_{2}, \ldots, p_{5}\right)$ in $\mathcal{T}$. Let $T^{i}$ denote a tree associated with $p_{i}$. Then, we can see that $\left|T^{5}\right| \leq \frac{3}{4}\left|T^{1}\right|$ always holds. Therefore, the length of a path from the root of $\mathcal{T}$ to any leaf is $5\left(\log _{(4 / 3)} n+1\right)$.

From this lemma, it is seen that TREE-BISECTION works in polynomial time. Now, we show our main result.

Theorem 2 TREE-BISECTION computes in polynomial time an SEOTG of size $O\left(m^{*} n^{5 / 6}\right)$ for a given rooted ordered tree $T$, where $m^{*}$ is the size of the smallest EOTG for $T$, and $n=|T|$.

Proof. As in [3], it is enough to bound the number of non-isomorphic subtrees generated by TREE-BISECTION because a production rule of size at most 4 is generated per subtree.

First, we count the number of subtrees generated by TREE-BISECTION whose sizes are greater than $n^{\alpha}$, where $\alpha$ is a constant to be determined later. From Lemma 3, the number of such subtrees generated by recursive calls at depth $d$ is $(n-1) / n^{\alpha}<n^{1-\alpha}$. Since the maximum depth is $O(\log n)$ from Lemma 4, the number of subtrees whose sizes are greater than $n^{\alpha}$ is $O\left(n^{1-\alpha} \log n\right)$. 
Next, we count the number of non-isomorphic subtrees of size at most $n^{\alpha}$. Recall that the Euler string of any tagged tree has a form of $s_{1} x s_{2}$, where each $s_{1}$ and $s_{2}$ is a substring of $e s\left(T_{0}\right)$. Therefore, the number of non-isomorphic subtrees of size $k$ is bounded by

$$
2 m^{*}(2 k-2)+\sum_{k_{1}=1}^{(2 k-2)-1}\left(2 m^{*} k_{1}\right)\left(2 m^{*}\left((2 k-2)-k_{1}\right)\right) \leq c_{1}\left(m^{*}\right)^{2} k^{3}
$$

from Lemma 2 since the length of the Euler string of a subtree of size $k$ is $2(k-1)$, where $c_{1}$ is some constant. Then, the number of non-isomorphic subtrees of size at most $n^{\alpha}$ is $\sum_{k=1}^{n^{\alpha}} c_{1}\left(m^{*}\right)^{2} k^{3} \leq c_{2} \cdot\left(m^{*}\right)^{2} \cdot n^{4 \alpha}$.

By summing up these two numbers, the total number of non-isomorphic subtrees generated by TREE-BISECTION is $O\left(\left(m^{*}\right)^{2} \cdot n^{4 \alpha}+n^{1-\alpha} \log n\right)$. Letting $\alpha=1 / 6$ and assuming that $m^{*}$ is $O\left(n^{(1 / 6)}\right)$, we can see that the total number of non-isomorphic subtrees is

$$
O\left(m^{*} \cdot n^{(1 / 6)} \cdot n^{(4 / 6)}+n^{(5 / 6)} \log n\right)=O\left(m^{*} \cdot n^{(5 / 6)}+n^{(5 / 6)} \log n\right) .
$$

Since $m^{*} \cdot n^{(5 / 6)} \geq n$ holds for $m^{*} \geq n^{1 / 6}$ and $m^{*}$ is $\Omega(\log n)$, the number of non-isomorphic subtrees generated by TREE-BISECTION is $O\left(m^{*} n^{5 / 6}\right)$.

TREE-BISECTION can be modified for compression of rooted unordered trees of bounded degree (i.e., rooted unordered trees in which the number of children of each node is bounded by a constant $H$ ). In this case, the definitions of grammars remain the same except that we do not distinguish the orders of children. For example, we do not distinguish type (IIIA) rules from type (IIIB) rules. Let EUTG (elementary unordered tree grammar) and SEUTG (simple elementary unordered tree grammar) be the resulting grammars corresponding to EOTG and SEOTG, respectively.

For compression of unordered trees, we modify TREE-BISECTION as follows.

- In partition of a non-tagged tree $T$, we partition $T_{2}$ into $T_{2+i}=s u b\left(v, w_{i}\right)(i=1, \ldots, h)$.

- In partition of a tagged tree $T$, we also partition $T_{2}$ into $T_{2+i}=\operatorname{sub}\left(v, w_{i}\right)(i=1, \ldots, h)$.

- We replace the subtree isomorphism test for ordered trees with one for unordered trees.

Let UNORDERED-TREE-BISECTION denote the resulting algorithm. It is to be noted that UNORDERED-TREE-BISECTION does not necessarily output an SEUTG, instead it may output an EUTG because production rules of size $2 H$ may be generated. Since $T_{2}$ is uniquely determined from $T$ and is uniquely decomposed into $T_{2+i} \mathrm{~s}$, the decomposition of an input 
tree is independent of the ordering of children. That is, the same unordered grammar is always obtained if isomorphic trees are given.

Theorem 3 UNORDERED-TREE-BISECTION computes in polynomial time an EUTG of size $O\left(m^{*} n^{5 / 6}\right)$ for a given rooted unordered tree $T$ of bounded degree, where $m^{*}$ is the size of the smallest EUTG for $T$, and $n=|T|$.

Proof. It is straight-forward to verify that Proposition 2, Lemma 3 and Lemma 4 hold for UNORDERED-TREE-BISECTION. Since it is known that isomorphism of unordered trees can be tested in linear time [4], the algorithm works in polynomial time.

Next, we consider the approximation ratio. Though we do not distinguish the orders of children in grammars or input trees, we can assume that an input tree is generated by a minimum size EOTG and then the orders of children are ignored. Therefore, the number of non-isomorphic unordered subtrees of size $k$ is bounded by $c_{1}\left(m^{*}\right)^{2} k^{3}$ as in the proof of Theorem 2 and thus the total number of non-isomorphic subtrees produced by the algorithm is $O\left(m^{*} \cdot n^{(5 / 6)}+n^{(5 / 6)} \log n\right)$, which is $O\left(m^{*} \cdot n^{(5 / 6)}\right)$ because $m^{*}$ is $\Omega(\log n)$. Since the size of each generated production rule is bounded by $2 H$ and $H$ is assumed to be a constant, the size of the resulting grammar remains $O\left(m^{*} \cdot n^{(5 / 6)}\right)$.

\section{Acknowledgement}

The author would like to thank Morihiro Hayashida and Yang Zhao for helpful discussions.

\section{References}

[1] T. Akutsu, A relation between edit distance for ordered trees and edit distance for Euler strings, Information Processing Letters 100 (2006) 105-109.

[2] G. Busatto, M. Lohrey, S. Maneth, Efficient memory representation of XML document trees, Information Systems 33 (2008) 456-474.

[3] M. Charikar, E. Lehman, D. Liu, R. Panigrahy, M. Prabhakaran, A. Sahai, A. Shelat, The smallest grammar problem, IEEE Transactions on Information Theory 51 (2005) 2554-2576.

[4] Y. Dinitz, A. Itai, M. Rodeh, On an algorithm of Zemlyachenko for subtree isomorphism, Information Processing Letters 70 (1999) 141-146. 
[5] J. C. Kieffer, E-H. Yang, Grammar-based codes: A new class of universal lossless source codes, IEEE Transactions on Information Theory 46 (2000) 737-754.

[6] W. Rytter, Application of Lempel-Ziv factorization to the approximation of grammarbased compression, Theoretical Computer Science 302 (2003) 211-222.

[7] S. Murakami, K. Doi, A. Yamamoto, Finding frequent patterns from compressed treestructured data, Proc. 11th Int. Conf. Discovery Science (2008) 284-295.

[8] H. Sakamoto, S. Maruyama, T. Kida, S. Shimozono, A space-saving approximation algorithm for grammar-based compression, IEICE Transactions on Information and Systems 92-D (2009) 158-165.

[9] K. Yamagata, T. Uchida, T. Shoudai, Y. Nakamura, An effective grammar-based compression algorithm for tree structured data, Proc. 13th Int. Conf. Inductive Logic Programming (2003) 383-400. 